\title{
Enhancement of Deep Epileptiform Activity in the EEG via 3-D Adaptive Spatial Filtering
}

\author{
Donna-Maree Ward, Member, IEEE, Richard D. Jones,* Senior Member, IEEE, \\ Philip J. Bones, Senior Member, IEEE, and Grant J. Carroll
}

\begin{abstract}
The detection of epileptiform discharges (ED's) in the electroencephalogram (EEG) is an important component in the diagnosis of epilepsy. However, when the epileptogenic source is located deep in the brain, the ED's at the scalp are often masked by more superficial, higher-amplitude EEG activity. A noninvasive technique which uses an adaptive "beamformer" spatial filter has been investigated for the enhancement of signals from deep sources in the brain suspected of containing ED's. A forward three-layer spherical model was used to relate a dipolar source to recorded signals to determine the beamformer's spatial response constraints. The beamformer adapts, using the least-mean-squares (LMS) algorithm, to reduce signals from sources distant to some arbitrarily defined location in the brain. The beamformer produces three outputs, being the orthogonal components of the signal estimated to have arisen at or near the assumed location.
\end{abstract}

Simulations were performed by using the same forward model to superimpose realistic ED's on normal EEG recordings. The simulations show the beamformer's ability to enhance signals emanating from deep foci by way of an enhancement ratio (ER), being the improvement in signal-to-noise ratio (SNR) to that observed at any of the scalp electrodes. The performance of the beamformer has been evaluated for 1) the number of scalp electrodes, 2) the recording montage, 3 ) dependence on the background EEG, 4) dependence on magnitude, depth, and orientation of epileptogenic focus, and 5) sensitivity to inaccuracies in the estimated location of the focus.

Results from the simulations show the beamformer's performance to be dependent on the number of electrodes and moderately sensitive to variations in the EEG background. Conversely, its performance appears to be largely independent of the amplitude and morphology of the ED. The dependence studies indicated that the beamformer's performance was moderately dependent on eccentricity with the ER increasing as the dipolar source and the beamformer were moved from the center to the surface of the brain (1.51-2.26 for radial dipoles and 1.17-2.69 for tangential dipoles). The beamformer was also moderately dependent on variations in polar or azimuthal angle for radial and tangential dipoles. Higher ER's tended to be seen for locations between electrode sites.

The beamformer was more sensitive to inaccuracies in both polar and azimuthal location than depth of the dipolar source. For polar locations, an ER $>1.0$ was achieved when the beamformer was located within $\pm 25^{\circ}$ of a radial dipole and $\pm 35^{\circ}$ of a

Manuscript received September 2, 1997; revised January 7, 1999. Asterisk indicates corresponding author.

D.-M. Ward and P. J. Bones are with the Department of Electrical and Electronic Engineering, University of Canterbury, Christchurch, New Zealand.

*R. D. Jones is with the Department of Medical Physics and Bioengineering, Christchurch Hospital, Christchurch, New Zealand. He is also with the Department of Medicine, Christchurch School of Medicine, Christchurch, New Zealand (e-mail: richard.jones@chmeds.ac.nz).

G. J. Carroll is with the Department of Neurology, Christchurch Hospital, Christchurch, New Zealand.

Publisher Item Identifier S 0018-9294(99)03986-5. tangential dipole. Similarly, angular ranges of $\pm 37.5^{\circ}$ and $\pm 45^{\circ}$, respectively, for inaccuracies in azimuthal locations. Preliminary results from real EEG records, comprising 12 definite or questionable epileptiform events, from four patients, demonstrated the beamformer's ability to enhance these events by a mean $100 \%$ $(52 \%-215 \%)$ for referential data and a mean $104 \%(50 \%-145 \%)$ for bipolar data.

Index Terms-Adaptive spatial filtering, electroencephalogram (EEG), epilepsy.

\section{INTRODUCTION}

$\mathbf{T}$ HE detection of epileptiform discharges (ED's) — spikes and sharp waves - occurring in the electroencephalogram (EEG) between seizures is an important component in the diagnosis of epilepsy. When an epileptogenic source is located near the surface of the brain, the corresponding EEG will contain characteristic ED's which are strongly indicative of epilepsy. However, in the case of an epileptogenic source located deep in the brain, these ED's may be sufficiently masked by the more superficial background EEG as to be difficult to detect by the electroencephalographer (EEGer), such as is often the case in temporal lobe epilepsy. Currently, the only means of enhancing ED's located deep in the brain is by invasive electrophysiological techniques, such as depth electrodes, sphenoidal electrodes and electrocorticography, or by very expensive techniques such as magnetoencephalography.

Lopes da Silva et al. used inverse autoregressive filtering to effectively enhance nonstationarities, including ED's, in the EEG [1]. This became the central component of a spike detector which had an understandably high false-detection rate in all but very artifact-free EEG's. Similarly, James et al. used multireference adaptive noise cancelling to enhance nonstationarities by means of a multilayer perceptron artificial neural network [2]. This was proposed as a precursor in a multistage spike detection system with the aim of increasing the overall sensitivity to ED's.

Van Veen et al. [3], Spencer et al. [4], and van Drongelen et al. [5] have applied an adaptive three-dimensional spatial filtering technique to help detect and localize epileptogenic sources in the brain. This technique is commonly referred to as 'beamforming' due to the origins of the technique as used in radar, sonar, geophysical and astrophysical exploration applications [6]. The performance of the beamforming technique, when applied to EEG records, relies on the existence of $a$ priori clinical information indicating the approximate location 


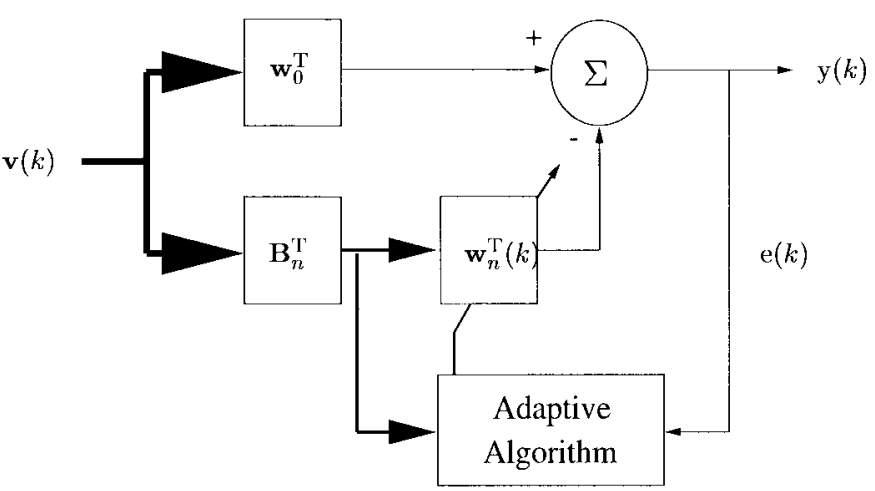

Fig. 1. The adaptive linearly constrained minimum variance (LCMV) beamformer with a nonadaptive section having a fixed weight vector $\boldsymbol{w}_{0}$ and an adaptive section composed of an adaptive weight vector $\boldsymbol{w}_{n}$ and blocking matrix $\boldsymbol{B}_{n}$. The blocking matrix prevents the adaptive portion from cancelling any signals propagating from the location of interest while allowing the passage of the other signals from other locations. An unconstrained adaptive algorithm is used to minimize the output power.

of a possible epileptogenic source. This information can be used by the beamformer to enhance activity being generated in the vicinity of that location or, more precisely, to attenuate EEG and artifacts emanating from areas elsewhere in and around the brain.

This paper presents a comprehensive investigation of the performance characteristics of the beamforming technique when applied to enhancement of ED's in the EEG. The primary investigation was carried out by way of simulated dipolar ED's at specified sites in the brain. This allowed us to determine the dependence of the beamformer's performance on number of electrodes, the recording montage, and the magnitude, location and orientation of the dipolar source within the brain, and to determine the sensitivity of the beamformer to inaccuracies in the estimate of location of the dipolar source. The paper also presents preliminary results demonstrating the enhancement of ED's in real EEG data.

\section{The BeAmforming TeChnique}

The beamformer used to process EEG signals is depicted in Fig. 1. This particular design is known as either an adaptive linearly constrained minimum variance (LCMV) beamformer or a generalized sidelobe canceller (GSC) [4], [6], [7]. It has the effect of preserving the signal arising from the chosen source location while minimizing the contributions from other sources.

In the EEG case, there are 16 channels of input $\boldsymbol{v}(k)$ to the beamformer and a single output channel $\mathrm{y}(k)$. Essentially, Fig. 1. depicts two beamformers operating in unison. The first beamformer, represented by the top branch in Fig. 1, is a nonadaptive section which contains a set of fixed weights $w_{0}$ which implement a spatial filter on the input EEG data. Hence, the output signal produced from this branch is composed of background noise, which has been reduced compared to that seen at any of the input signals $\boldsymbol{v}(k)$, and the signal of interest, that is, the ED. Alternatively, the second beamformer (lower branch in Fig. 1) is an adaptive section comprising a fixed blocking matrix $\boldsymbol{B}_{n}$ and a set of variable weights $\boldsymbol{w}_{n}(k)$.

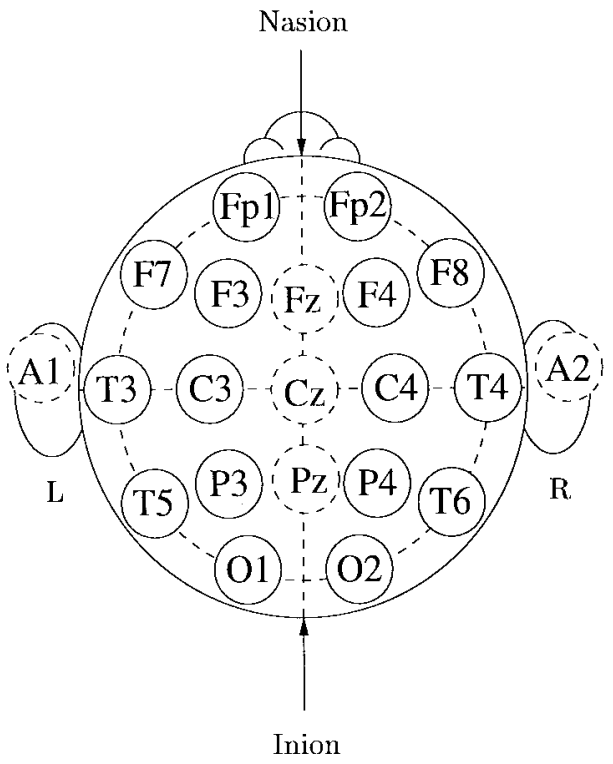

Fig. 2. The 10-20 International System of electrode placement.

The role of the blocking matrix is to block signals arising from the location of interest while allowing signals from other locations to pass through as the reduced dimensional vector $\boldsymbol{u}(k)$. The least mean squares (LMS) algorithm is used to update the set of weights $\boldsymbol{w}_{n}(k)$. The output of the adaptive branch is essentially a model of the background noise plus a slight contribution of the signal of interest. The scalar result of subtracting the adaptive section output from the nonadaptive section output serves to cancel signals from outside the location of interest. Consequently, at the output $\mathrm{y}(k)$ we obtain a single channel representing an estimate of the signal emanating from an equivalent dipolar source at a chosen location and orientation. Three such beamformers can be combined to resolve the source orientation, one for each of the three orthogonal directions $x, y$, and $z$. Further details of the calculation of $w_{0}, B_{n}$, and $\boldsymbol{w}_{n}$ are given by [3]-[6].

\section{Simulations}

Data for the simulation studies was obtained from routine EEG recordings at Christchurch Hospital. Sixteen channels of EEG were recorded on the ipsilateral-ears referential montage from electrodes placed according to the 10-20 International System [8] (Fig. 2). Each channel of the recorded EEG was lowpass filtered at $70 \mathrm{~Hz}(100 \mathrm{~dB} / \mathrm{dec})$, sampled at a rate of 200/s, and digitized to 12-bit precision.

ED's and normal background EEG were extracted from epileptiform and normal EEG recordings respectively to facilitate controlled investigation of the beamformer's performance and characteristics on simulated data. Each simulated epoch of data was arbitrarily chosen to be of 4-s (800 samples) duration since the beamformer required of the order of $2 \mathrm{~s}$ (400 samples) to adapt.

EEG recordings from patients with focal epilepsy were viewed by an EEGer for definite ED's. These ED's were then 


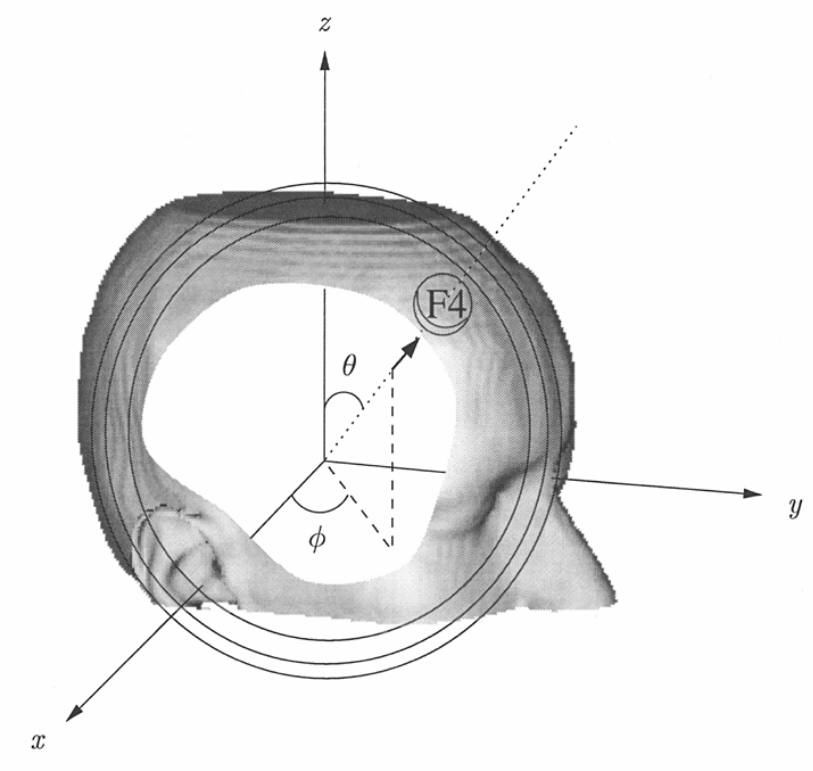

Fig. 3. Coordinate system used with location and direction of deep epileptogenic source given as $\theta=45^{\circ}, \phi=45^{\circ}$, and $e=0.5$. Origin of coordinates is $(0,0,0)$.

extracted from a single channel of the recorded EEG. The ED's were selected to represent the amplitude and morphology of ED's to be used as deep dipolar foci in simulation studies. The ED's selected included both monophasic and biphasic ED's of variable duration but were typically less than $140 \mathrm{~ms}$. The peak of the extracted ED was sited at 700 samples and padded with zeros to 800 samples. For all simulation studies, one monophasic and one biphasic ED, extracted from different patients, were each superimposed upon five background EEG records from different patients to yield ten sets of EEG data.

The isolated ED's were used as the amplitudes of simulated current dipoles at arbitrary fixed locations and orientations in the head. The location is specified by polar angle $\theta$, azimuthal angle $\phi$, and eccentricity $e$ (i.e., normalized depth of the source). A forward three-layered inhomogeneous and isotropic spherical model [9] was applied to determine the potential distribution on the surface of the scalp at the 16 electrode positions given in Fig. 2. The model consisted of three concentric spheres representing the scalp, skull, and brain (Fig. 3). The radii of these were 92, 85, and $80 \mathrm{~mm}$, respectively. The conductivity of the scalp and brain were both set to $0.33 \mathrm{~m}^{-1}$ while that of the skull was $0.0042 \mathrm{~m}^{-1}$. This gave a ratio of conductivity between the brain and skull of 80 [10].

This forward model was used to derive a transfer matrix $G$ $(16 \times 3)$ such that

$$
\boldsymbol{v}=\boldsymbol{G} \boldsymbol{q}
$$

where $\boldsymbol{q}(3 \times 1)$ represents the Cartesian components of a unit dipole at the chosen location and orientation and $\boldsymbol{v}(16 \times 1)$ is the set of electrode potentials arising from the dipolar source. For simulations the amplitude of the dipolar source was then modulated by a scalar amplitude $s_{\mathrm{ED}}(k)$, based on an actual recorded $\mathrm{ED}$, so that

$$
\boldsymbol{v}(k)=\boldsymbol{G} \boldsymbol{q} s_{\mathrm{ED}}(k) .
$$

The 16 channels of voltage determined at each electrode (with respect to reference electrodes A1 and A2) were then superimposed upon one of several normal background EEG recordings to yield multichannel scalp EEG $v(k)(16 \times 800)$ containing simulated spikes from dipoles located anywhere in the brain. This represented the EEG record to be input to the beamformer.

Different recording montages can be handled by the beamformer by premultiplying the input to the beamformer by an appropriate montage matrix $M$. The same forward transfer matrix $\boldsymbol{G}$ (for the chosen dipole position) was also used to derive the set of fixed weights $w_{0}$ and the blocking matrix $B_{n}$ for the beamformer.

In addition to visualizing the beamformer's output $\mathrm{y}(k)$ in any coordinate or resultant direction, we use the forward model to project the beamformer output to the surface of the scalp at standard 10-20 electrode sites and compute corresponding referential or bipolar channel signals as required.

\section{Measures of Performance}

In order to quantify improvements in SNR due to the beamformer, we define an enhancement ratio ER as

$$
\mathrm{ER}=\frac{\mathrm{SNR}_{o j}}{\mathrm{SNR}_{i j}}
$$

where $\mathrm{SNR}_{o j}$ is the inline beamformer output signal, $\mathrm{SNR}_{i j}$ is the input signal, and $j$ corresponds to the input channel containing the most prominent ED, that is, the highest $\mathrm{SNR}_{i j}$, where $j=1, \cdots, 16$. The "inline beamformer" signal defines the optimal case, in which the beamformer is placed at the same orientation as that of the dipolar source. Both $\mathrm{SNR}_{o j}$ and $\mathrm{SNR}_{i j}$ are defined as the ratio of the RMS amplitude of five samples $(25 \mathrm{~ms})$, centered on the peak of the spike, to the RMS amplitude of the background, also centered on the peak of the spike, over a 1-s interval. In all simulations the ER was determined by the inline beamformer. However, in the case of the projected output, an ER based on a singlechannel comparison is inadequate since the epileptiform event is enhanced to varying degrees on several channels. Therefore, a global ER performance measure is required. We have chosen to use the mean enhancement ratio $\overline{\mathrm{ER}}$ calculated from the ER for each of the 16 channels. However, because estimates of SNR become inaccurate for low signal levels, input channels with very low SNR's can lead to erroneous ER's and, hence, an inaccurate $\overline{\mathrm{ER}}$. To overcome this, we have chosen to use an arbitrary (based on observations made with an EEGer) SNR threshold of 0.7. Thus, any channel (input or output) which has a SNR $<0.7$ is excluded from the calculation of $\overline{\mathrm{ER}}$.

\section{Simulation Results}

To determine the characteristics of the beamformer in relation to the enhancement of ED's, we have undertaken a range of simulation studies. 


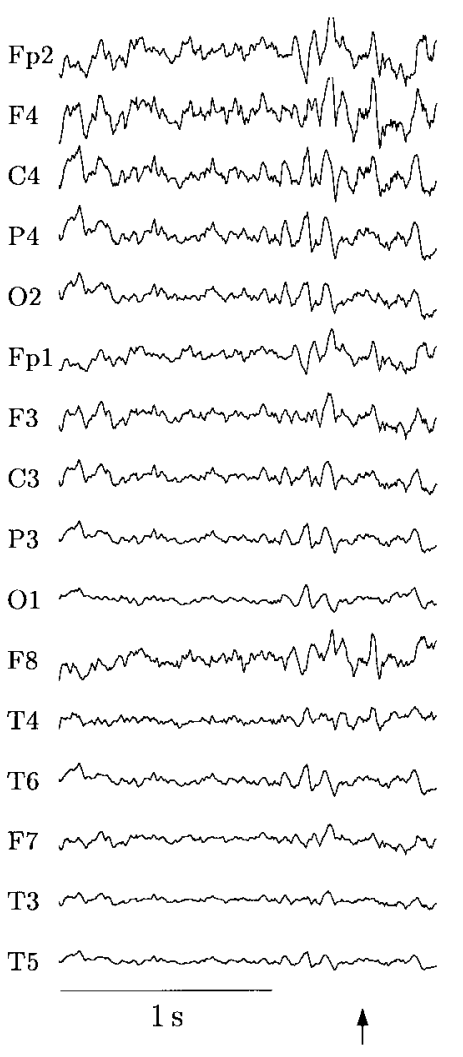

(a)

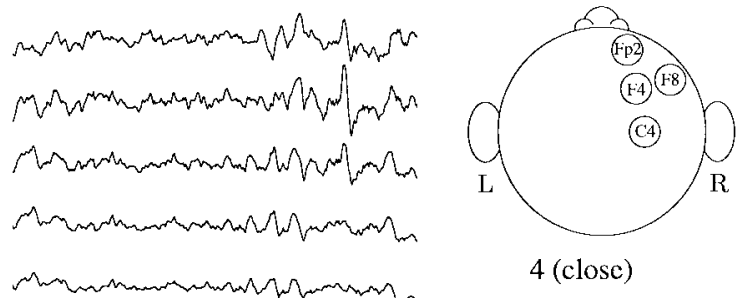

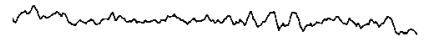
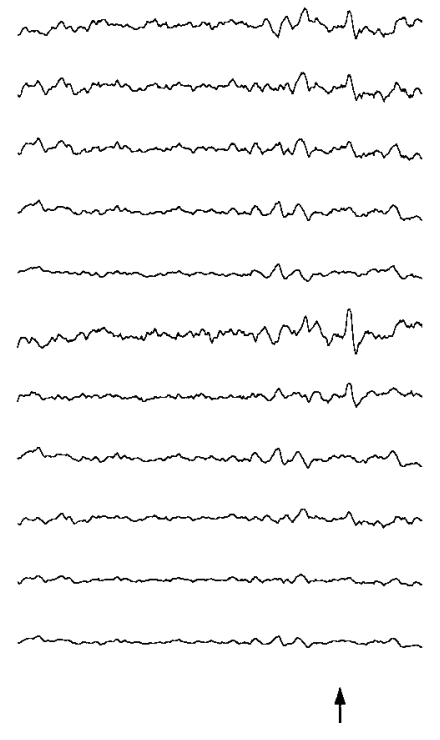

(c)

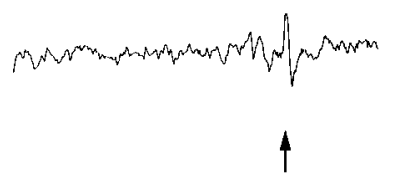

(b)

Fig. 4. An example of beamformer in action: (a) EEG record input to the beamformer for the case of the dipole placed at location shown in Fig. 3, (b) inline adapted output signal. All waveforms are of 2-s duration and have been normalized to the maximum amplitude of the ED in each record-channel F4 in both (a) and (b), and (c) projected beamformer output to the surface. It is important to recognize that the normalization results in there being no apparent enhancement of the magnitude of the most prominent ED (i.e., at F4 in this case).

\section{A. Enhancement of ED's}

An example of the beamformer's ability to enhance ED's is shown in Fig. 4. In this case, the ED source is represented by a radially oriented current dipole at $\theta=45^{\circ}, \phi=45^{\circ}$, and $e=0.5$ as illustrated in Fig. 3 (i.e., directly under F4). The latter $2 \mathrm{~s}$ of the 4-s segment of 16 channels of EEG input to the beamformer is shown in Fig. 4(a) in which the arrows indicate the position of a biphasic ED in the EEG record. In this particular case, the maximum simulated ED signal in the raw EEG occurred at electrode F4. Processing the data by the inline beamformer produced the output in Fig. 4(b) in which the relative amplitude of the ED has clearly been enhanced relative to any of the surface channels in Fig. 4(a). An ER of 1.73 was obtained in this case (with respect to F4). The projected output is shown in Fig. 4(c). Here, the enhancement of the ED on several channels is clearly evident with a $\overline{\mathrm{ER}}$ of 2.91 .

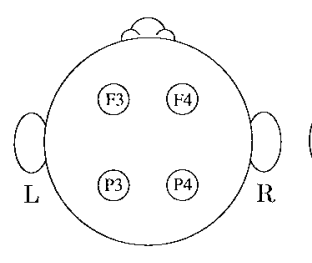

4 (far)

Fig. 5. Close and far sets of electrodes used to evaluate the sensitivity of the beamformer to the number and placement of electrodes.

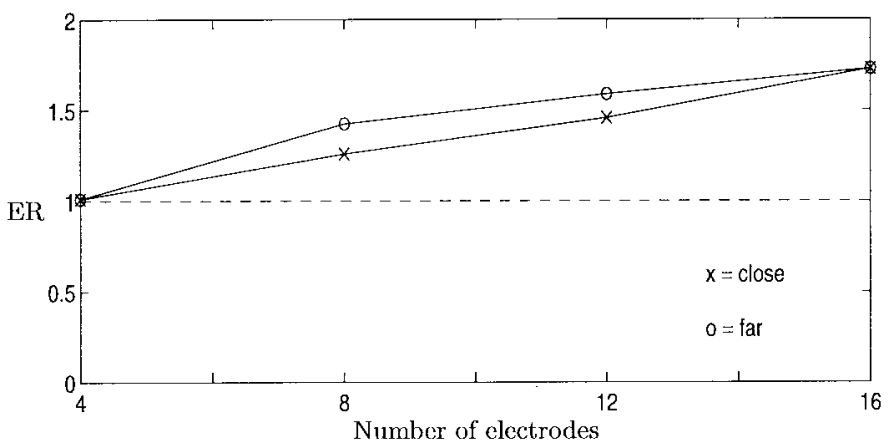

Fig. 6. Dependence of the beamformer on the number and spacing of electrodes.

\section{B. Dependence}

Simulations were performed to determine the extent to which the beamformer's performance is dependent on: number and location of scalp electrodes; recording montage; background EEG; magnitude, location and direction of the dipolar source. Thus, "dependence" represents the effect on the enhancement ratio (ER) of variations in any of these parameters when the beamformer is placed at exactly the same location and orientation as the dipole. The dipolar source and the beamformer were moved together to each new position. Radial and tangential dipoles were used in the simulations, although results are presented for radial dipoles only.

1) Dependence of Beamformer on Number and Location of Electrodes: Test sets of 4, 8, 12, and 16 electrodes, arranged in both close and more spread out arrays according to the standard 10-20 International System were investigated (Fig. 5).

A radially oriented dipole at $\theta=45^{\circ}, \phi=45^{\circ}$, and $e=0.5$ (Fig. 3) was selected to represent the dipolar source. Electrode F4 was closest to this dipole and hence was incorporated into each test set.

With the beamformer placed at the same location and with the same orientation as the dipole, the six arrays gave enhancements as shown in Fig. 6. As expected, the beamformer's performance improved as a function of the number 

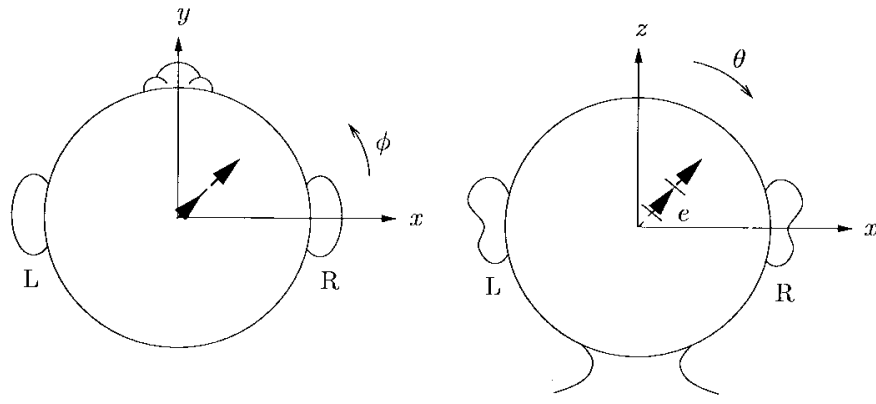

Top vicw

Back view

Fig. 7. Location and orientation of dipolar source for study of the dependence of ER on eccentricity of dipole and beamformer $\left(\theta=45^{\circ}, \phi=45^{\circ}\right)$.

of electrodes, with an ER of 1.01 for four closely-spaced electrodes increasing to 1.73 for all 16 electrodes. Small additional improvements were seen when the electrodes were spread out but only for the sets of eight and 12 electrodes. This slight superiority is presumed to be due to a more spread out set providing more spatial information to the beamformer.

2) Dependence of ER on the Recording Montage: The next investigation addressed the dependence of the beamformer on the recording montage used. This required beamforming using the standard ten ipsilateral-ears referentially recorded background EEG records (see Section III), reformatting each of these records into longitudinal-chains bipolar EEG records, and beamforming on these new records. In each case, the source was a radially oriented dipole (Fig. 3) with either a monophasic or a biphasic ED waveform. Differences in ER as large as $93 \%$ were found between the two montages for a given location and ED. However, overall, ED enhancement was reasonably similar with a mean ER of 1.47 (1.08-1.75) with the referential montage and a mean ER of 1.41 (1.06-1.67) with the bipolar montage.

3) Dependence of ER on ED and Background EEG: For this investigation, the standard ten data sets were used to assess the beamformer's performance. A radially oriented dipole at the location shown in Fig. 3 was selected to represent the dipolar source. The ER's were found to range from 1.13-1.75 for the monophasic ED and 1.10-1.73 for the biphasic ED. Thus, although only a limited investigation, ER appears to be relatively insensitive to ED morphology but moderately sensitive to background EEG.

4) Dependence of ER on Epileptiform Discharge Magnitude: Studies with monophasic and biphasic sources superimposed upon noisy background EEG, demonstrated that the ER was virtually independent of the amplitude of the ED. Variations ranged from $0.3 \%-3.9 \%$ for radially oriented dipoles and $1.5 \%-4.7 \%$ for tangentially oriented dipoles.

5) Dependence of ER on Eccentricity of Dipole and Beamformer: To determine the dependence of ER on eccentricity, both the polar angle $\theta$ and the azimuthal angle $\phi$ were held constant $\left(\theta=45^{\circ}, \phi=45^{\circ}\right)$ as a radially oriented dipolar source and the beamformer were moved along a radial line (Fig. 7).

Beamforming at equally spaced locations along the radial line using the standard ten data sets produced an average ER

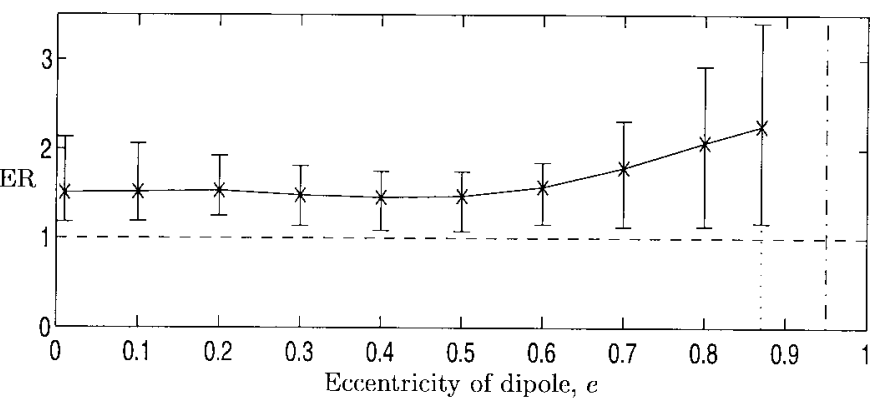

Fig. 8. Dependence of ER on eccentricity $e$ of a radial dipole for the case shown in Fig. 7. The average and range of ER's for the standard ten data sets are shown at each value of $e$. The brain-skull interface is shown at $e=0.87$ and the skull-scalp interface at $e=0.95$.

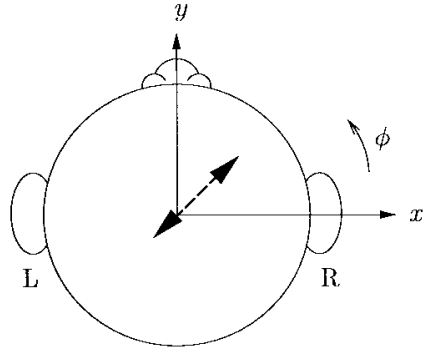

Top view

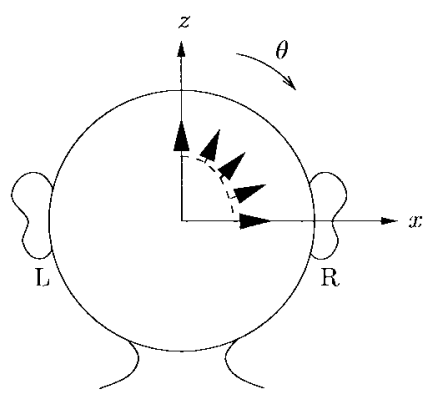

Back view
Fig. 9. Location and orientation of dipolar source for study of the dependence of ER on polar location of dipole and beamformer $\left(\phi=45^{\circ}, e=0.5\right)$.

which increased from 1.51-2.26 for radial dipoles (Fig. 8) and from 1.17-2.69 for tangential dipoles as the dipolar source and the beamformer were moved toward the surface of the brain. Also, an increase in variance of ER can be seen in Fig. 8 as the dipolar source and the beamformer are moved superficially. This occurs since the beamformer weights much higher those electrodes closest to the dipolar source when the assumed dipole location is near the surface than it does for the deeper assumed location. As a result, any variation in the background EEG in those electrodes is amplified and the variation between EEG epochs is emphasized, leading to either increased enhancement (higher ER) or decreased enhancement (lower ER).

6) Dependence of ER on Polar Angle of Dipole and Beamformer: In this case, both the azimuthal angle $\phi$ and the eccentricity $e$ were kept constant, while the dipolar source was rotated about a polar angle $\theta$ to a new location (Fig. 9).

Beamforming for the dipole at each new location along the polar arc, using the same ten sets of input data as before, showed that the beamformer is not strongly dependent on polar angle location ( $E R=1.20-1.60$ ) (Fig. 10). It is worth noting that the maximum ER occurs when the dipole is not directly under an electrode (e.g., F4 at $\theta=45^{\circ}$ ) and will, in many cases, be maximal when furthest from any electrode.

However, beamforming using tangential dipoles displayed a slightly higher ER at $\theta=0^{\circ}$ in all cases and a wider range (ER $=1.12-2.92$ ). Overall, mean ER was 1.39 for radial dipoles and 1.84 for tangential dipoles. 


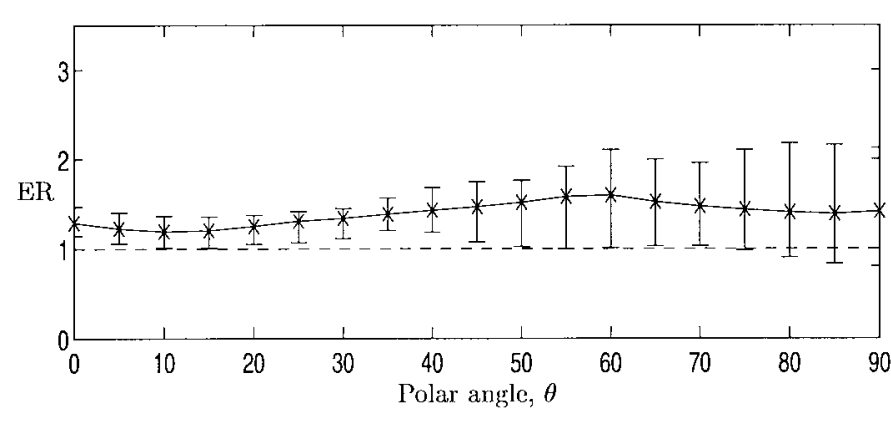

Fig. 10. Dependence of ER on polar angle $\theta$ of a radial dipole for the case shown in Fig. 9. For each location, the standard ten data sets were used.

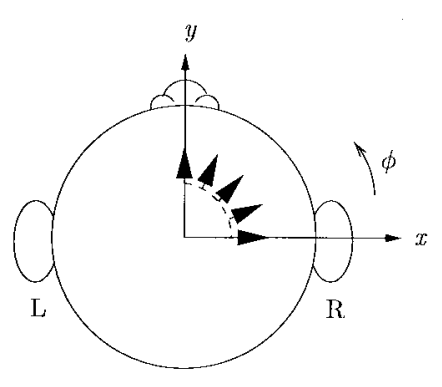

Top view

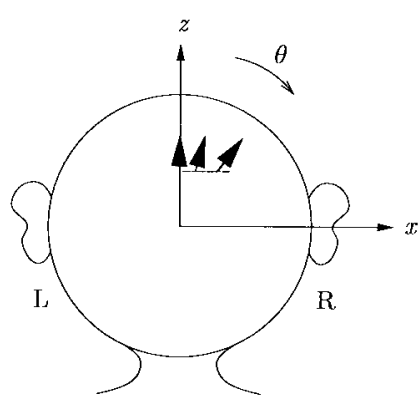

Back view
Fig. 11. Location and orientation of dipolar source for study of the dependence of ER on azimuthal location of dipole and beamformer $\left(\theta=45^{\circ}, e=0.5\right)$.

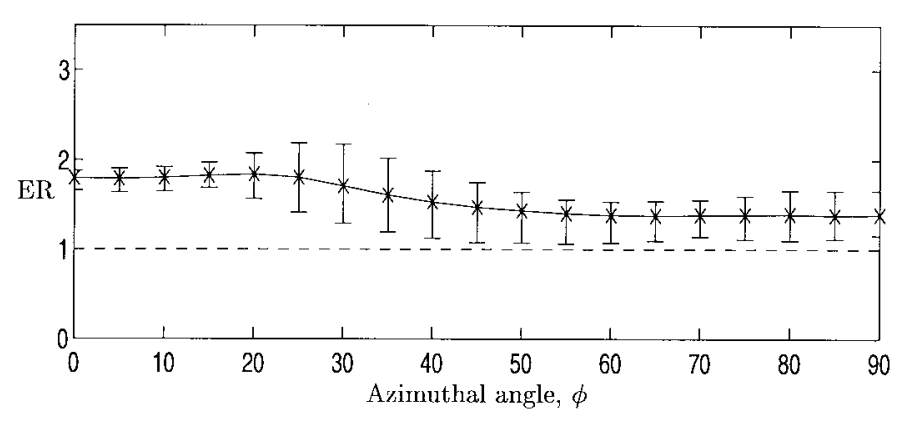

Fig. 12. Dependence of ER on azimuthal angle $\phi$ of a radial dipole for the case shown in Fig. 11. For each location, the standard ten data sets were used.

7) Dependence of ER on Azimuthal Location of Dipole and Beamformer: For this investigation, both the polar angle $\theta$ and the eccentricity $e$ were kept constant $\left(\theta=45^{\circ}, e=0.5\right)$, while the azimuthal angle $\phi$ of the dipolar source was rotated between $0^{\circ}$ and $90^{\circ}$ as depicted in Fig. 11 .

Beamforming for the dipolar source at equally spaced locations along the azimuthal arc, using the standard ten sets of input data, resulted in an ER as shown in Fig. 12, with a maximum of 1.84 at $\phi=22^{\circ}$ (i.e., between F4 and C4) for radial dipoles. The ER decreased on both sides of this maximum but remained greater than 1.0 at all locations along this azimuthal arc. Beamforming using tangential dipoles produced similar but flatter curves.

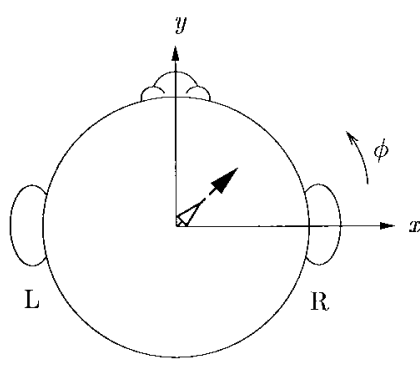

Top view

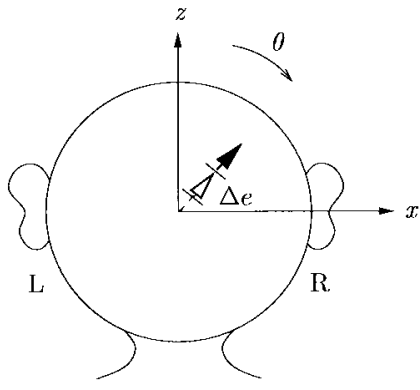

Back view
Fig. 13. Location and orientation of dipolar source for study of the sensitivity of beamformer to inaccuracies in eccentricity. Actual dipole (solid arrow) is located at $\theta=45^{\circ}, \phi=45^{\circ}$, and $e=0.5$ (Fig. 3). Estimated dipole is given by unfilled arrow.

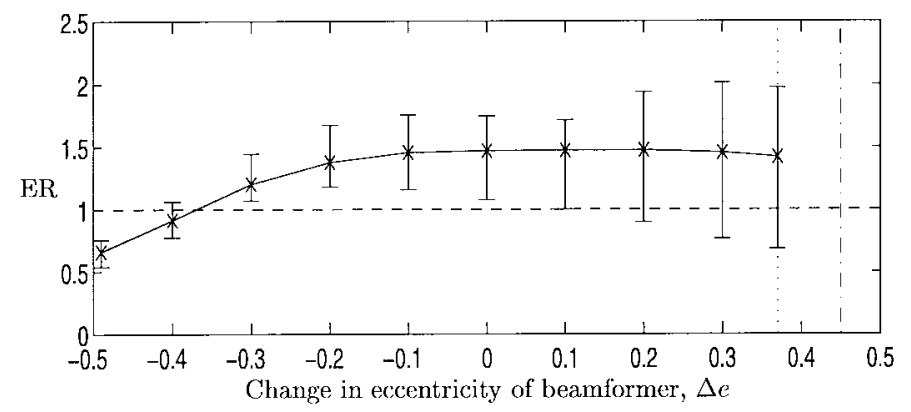

Fig. 14. Sensitivity of beamformer to inaccuracies in eccentricity $e$ of beamformer for the case shown in Fig. 13. The dipolar source was fixed at $e=0.5$. For each location, the standard ten data sets were used.

\section{Sensitivity}

Simulations were performed to determine the sensitivity of the beamformer's performance to inaccuracies in the assumed location of a dipolar source. In all cases, the actual dipole (solid arrow) was placed at a fixed location of $\theta=45^{\circ}$, $\phi=45^{\circ}$, and $e=0.5$ (Figs. 13, 15, and 17) and beamforming was undertaken on the standard ten different sets of input data.

1) Sensitivity of Beamformer to Inaccuracies in Depth: In this simulation, the location of the estimated dipole (unfilled arrow) was obtained by translation along the radial direction, a distance $\Delta e$ from the actual dipole (Fig. 13).

Beamforming at the location of the estimated dipole showed that the beamformer's performance is relatively insensitive to the depth of the estimated dipole until the estimated dipole gets near the center of the head $(e=0.3$ or $\Delta e=-0.2$ in Fig. 14). No enhancement was observed when the beamformer was located at $e<0.12$. As for the dependence studies, an increase in the variance of ER as the estimated dipole was moved toward the surface of the head can be seen in Fig. 14.

2) Sensitivity of Beamformer to Inaccuracies in Polar Location: In this simulation the beamformer was rotated in the $z$-axis through an angle $\Delta \theta$ with respect to the location of the actual dipole along the dashed arc in Fig. 15.

Beamforming at each new location produced Fig. 16. The ER was a maximum 1.48 at the location of the actual dipole and decreased with increasing polar separation, with 


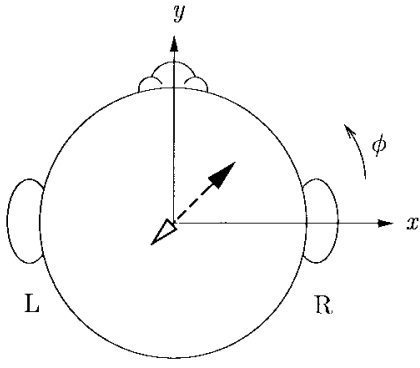

Top view

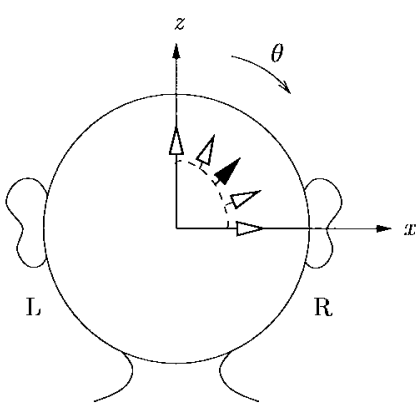

Back view
Fig. 15. Location and orientation of dipolar source for study of the sensitivity of beamformer to inaccuracies in polar location. Actual dipole (solid arrow) is located at $\theta=45^{\circ}, \phi=45^{\circ}$, and $e=0.5$ (Fig. 3). Estimated dipole is given by unfilled arrow.

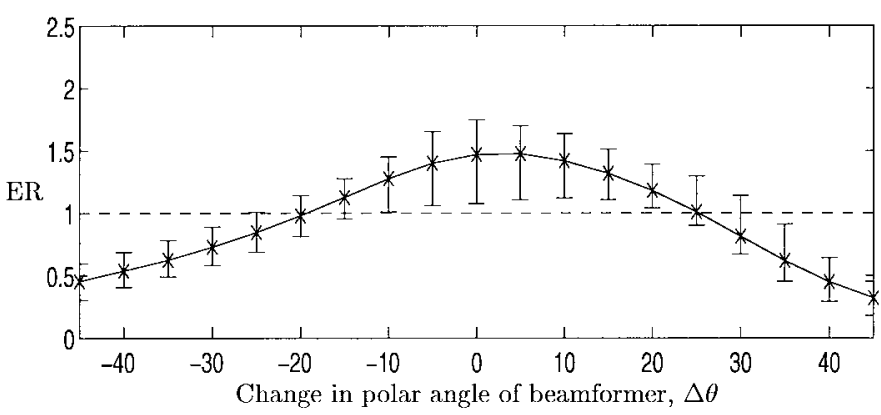

Fig. 16. Sensitivity of beamformer to inaccuracies in polar location $\theta$ of beamformer for the case shown in Fig. 15. For each location, the standard ten data sets were used.

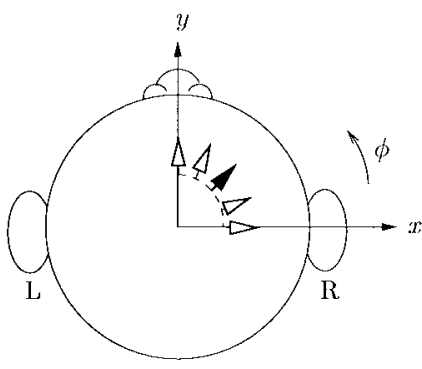

Top view

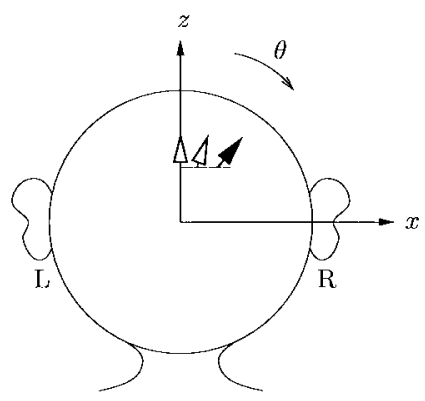

Back view
Fig. 17. Location and orientation of dipolar source for study of the sensitivity of beamformer to inaccuracies in azimuthal location. Actual dipole (solid arrow) is located at $\theta=45^{\circ}, \phi=45^{\circ}$, and $e=0.5$ (Fig. 3). Estimated dipole is given by unfilled arrow.

enhancement dropping to zero for inaccuracies in location of $\pm 20^{\circ}$ or greater. This indicates that the beamformer is substantially more sensitive to inaccuracies in the estimated polar location than of inaccuracies in the estimated eccentricity of the dipolar source.

3) Sensitivity of Beamformer to Inaccuracies in Azimuthal Location: The final simulation looked at the sensitivity of the beamformer to inaccuracies in azimuthal location of the dipolar source. Here, the beamformer was rotated through an angle $\Delta \phi$ with respect to the location of the actual dipole along the dashed arc in Fig. 17.

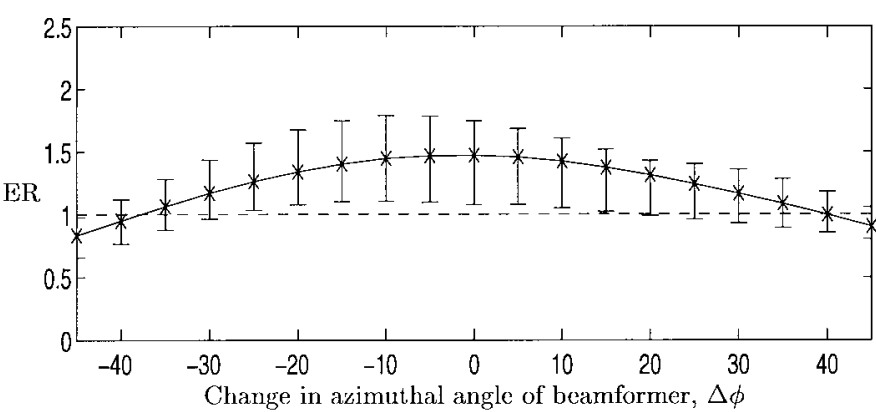

Fig. 18. Sensitivity of beamformer to inaccuracies in azimuthal angle $\phi$ of beamformer for the case shown in Fig. 17. For each location, the standard ten data sets were used.

Beamforming at each new location for the estimated dipole produced Fig. 18 in which ER was a maximum 1.48 at the exact location of the actual dipole and remained above 1.0 for $|\Delta \phi| \leq 37.5^{\circ}$.

\section{Clinical Evaluation}

The 20-min EEG's from four patients, all graded globally as containing epileptiform activity according to two independent EEGers, were used in a preliminary assessment of the beamformer's ability to enhance real ED's. From these EEG's, 12 events graded as definite or questionable focal epileptiform were selected for the evaluation. All 12 events were recorded using the ipsilateral-ears referential montage. These recordings were also subsequently reformatted into longitudinal-chains bipolar data for further processing by the beamformer.

For each patient, a priori information from the EEGer's report and visual cues in the EEG record were used to provide an estimate of the location of the epileptogenic source within the brain. The beamformer was then moved within a small volume $\left(60^{\circ} \times 60^{\circ} \times 0.4\right)$ around the estimated location to determine the position at which the beamformer achieved a maximum SNR. The SNR, calculated using the procedure described in Section IV, was found for the magnitude derived from the three orthogonal beamformer outputs, i.e. $\left(y_{x}^{2}+y_{y}^{2}+y_{z}^{2}\right)^{1 / 2}$.

Fig. 19(a) shows a typical EEG record from one of the patients containing a definite ED (based upon the context of the full EEG). Projecting the output from the beamformer onto the surface of the scalp produced Fig. 19(b) in which the enhancement of the ED is evident on several channels. In addition, the beamformer has filtered out much of the slower wave activity in the raw EEG. In this example, the beamformer was found to enhance this ED by a mean $215 \%$ (i.e., $\overline{\mathrm{ER}}=3.15$ ).

Fig. 20(a) shows the EEG record of Fig. 19(a) after reformatting into bipolar data, and Fig. 20(b) displays the corresponding projected beamformed output. Processing of the bipolar data resulted in a $\overline{\mathrm{ER}}$ of 1.67 , substantially lower than that for the corresponding referential case.

The referential and bipolar input and output records of all 12 definite or questionable ED's were randomized and shown to an EEGer to visually assess both the input and beamformed projected output EEG epochs by assigning a grade to each 
(a)

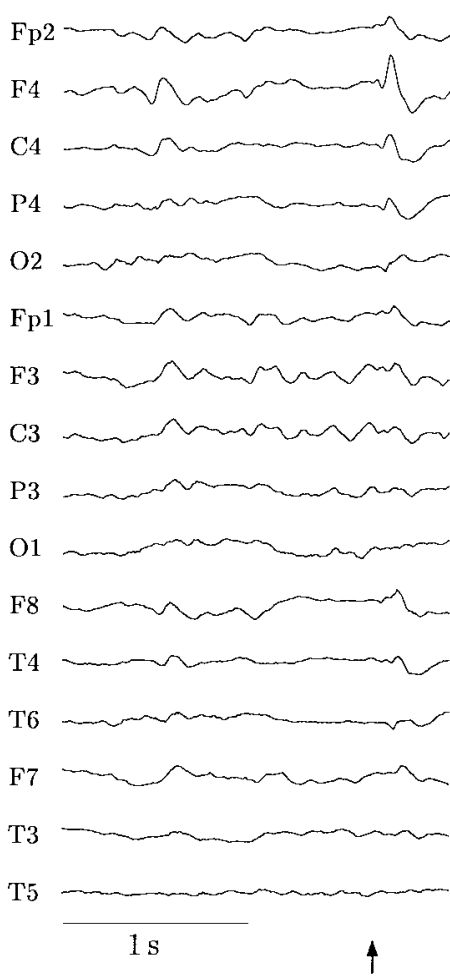

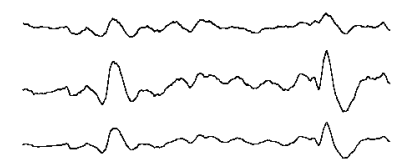
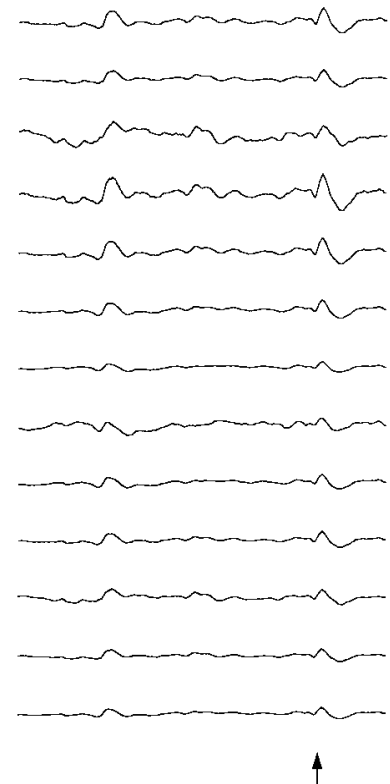

(b)

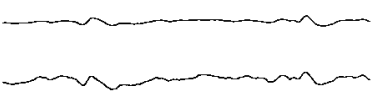

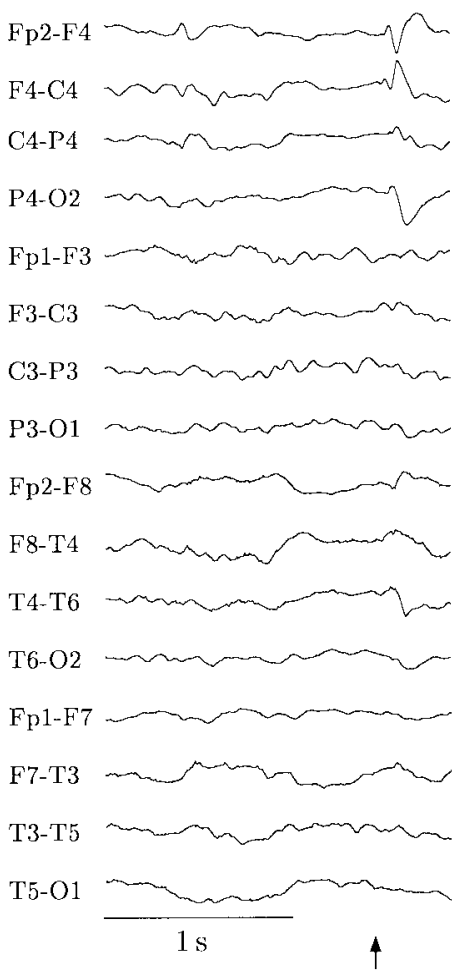

(a)

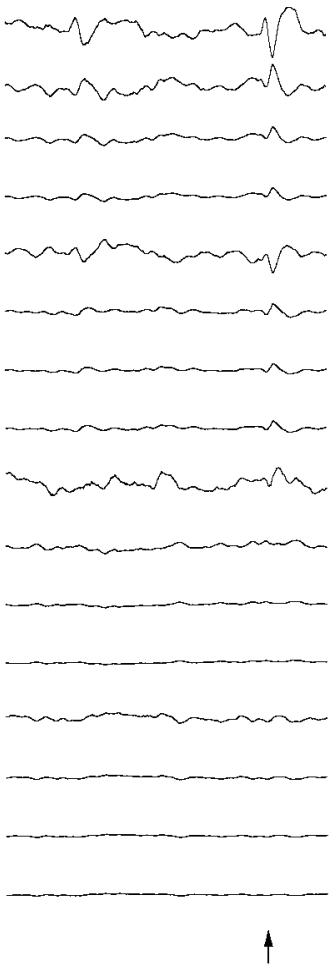

(b)
Fig. 19. (a) Ipsilateral-ears referential EEG record with a definite epileptiform event prior to input to the beamformer and (b) beamformer output projected onto the surface. All waveforms have been normalized to the maximum amplitude of the ED in each record-electrode F4 in both (a) and (b).

event. The EEGer classified each event as either definite, probable, possible, questionable, or nonepileptiform. Both the referential and bipolar versions of the ED in Figs. 19(a) and Fig. 20(a), respectively, were upgraded from a possible ED to a probable ED. The performance of the beamformer when applied to the 12 sets of referential and bipolar input and output records from the four patients is summarized in Table I.

On average, the beamforming technique is shown to enhance epileptiform events by a mean $100 \%(52 \%-215 \%)$ for referential data and a mean 104\% (50\%-145\%) for bipolar data.

\section{DISCUSSION}

The simulation results presented in this paper are a small selection from an extensive set. Each result has been confirmed by repeat simulations with different background EEG, different location and/or different ED. Investigations using both radially and tangentially oriented dipolar sources were performed although radially oriented dipoles have been presented in more detail.

Various approaches have been used by other authors for measuring the performance of beamforming techniques. A different approach from that implemented here defined the SNR as the average power of the source over all electrodes divided by the average noise power [3]-[5]. However, this approach can only be applied to the input signal of simulated
TABLE I

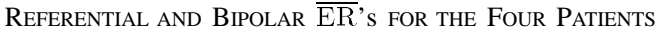

\begin{tabular}{c|c|c}
\hline \multirow{2}{*}{ Patient } & \multicolumn{2}{|c}{$\overline{\overline{\mathrm{ER}}}$} \\
\cline { 2 - 3 } & Referential & Bipolar \\
\hline \hline 1 & 2.29 & 2.45 \\
& 1.68 & 1.50 \\
& 3.15 & 1.67 \\
& 1.70 & 2.16 \\
\hline 2 & 2.18 & 1.96 \\
& 2.44 & 2.38 \\
\hline 3 & 1.52 & 1.68 \\
\hline 4 & 1.77 & 2.08 \\
& 1.85 & 2.20 \\
& 1.95 & 2.18 \\
& 1.94 & 2.18 \\
& 1.54 & 2.04 \\
\hline Mean & 2.00 & 2.04 \\
\hline \hline
\end{tabular}

data, whereas our method for determining the enhancement ratio ER, can be applied to both the input and the output signals of the simulations and real data. Also, some authors [3], [5] have measured the performance of this technique at equally-spaced intervals along horizontal cross-sectional grids throughout the spherical model (that is, along slices of constant $z$ ), whereas our method for determining ER is based on the 
signals observed on the surface of the scalp at the electrode sites. However, the inline ER determined in this paper does provide a relatively conservative measure of the performance of the beamformer. This point is illustrated in Fig. 4. where the visibility of the simulated ED in both the single adapted output and in the output projected onto the scalp is much improved although the ER of 1.73 implies only a modest enhancement. However, the converse is true when comparing Fig. 13(a) to (b) in which the projected output $\overline{\mathrm{ER}}$ is 3.15 .

The beamformer has been shown to be moderately dependent on depth, polar and azimuthal location of the dipolar source. However, the beamformer proved to be more sensitive to inaccuracies in both polar and azimuthal location than to depth of the dipolar source. This may provide a means of using the beamformer to improve precision of the localization of deep epileptogenic foci.

The computation involved in the beamforming technique is moderately demanding but, with further work, it is hoped that the beamforming method will be suitable for real-time use applications. Currently, a $4 \mathrm{~s}$ epoch of EEG takes $10 \mathrm{~s}$ to process on a Pentium 166-MHz processor.

The beamformer can be applied to different recording montages. The simulation results shown are for an ipsilateral-ears reference montage and a longitudinal-chains bipolar montage. Results using both montages reveal a similar $\overline{\mathrm{ER}}$. This has also been confirmed in the case of real EEG data.

In comparison with more realistic anatomical models of the head, the concentric-spherical model is reasonably accurate except in those regions of the head where the actual and model thickness vary the most [11], [12]. However, this is not a major concern in beamforming since the primary purpose of the beamformer is to detect rather than to locate sources and, thus, the emphasis in modeling is to simulate the approximate field rather than the exact field for a given source location and orientation. A more accurate head model may improve the performance of the beamformer but is likely to require more accurate source location information a priori than can realistically be expected. The effectiveness of incorporating a more accurate anatomical model of the head in place of the current spherical model is to be investigated.

Both the simulation and clinical results demonstrate that the beamforming technique has considerable potential for application in clinical neurophysiology. The beamforming technique can noninvasively enhance epileptiform activity from deep foci in the brain, such as in medial temporal lobe epilepsy, thereby minimizing the need for invasive electrophysiological investigations such as sphenoidal electrodes and provide an inexpensive alternative to electrophysiological techniques such as magnetoencephalography. It is considered that further improvements in enhancement may be obtained once the beamforming technique has been optimized. Possible improvement may result from the use of different adaptive algorithmic techniques (e.g., recursive least squares, normalized LMS, or block processing) and the application of additional constraints within the beamformer, for example. A more extensive clinical evaluation is also planned. Ultimately, the aim is to have the facility for the beamformer run in conjunction with real-time automated ED detection [13], [14].

\section{REFERENCES}

[1] F. H. Lopes da Silva, A. Dijk, and H. Smits, "Detection of nonstationarities in EEG's using autoregressive model-An application to EEG's of epileptics," in H. Kundel and G. Dolce, Eds., CEAN_Computerized EEG analysis. Stuttgart, Germany: Gustav Fischer Verlag, 1975.

[2] C. J. James, M. T. Hagan, R. D. Jones, P. J. Bones, and G. J. Carroll, "Multireference adaptive noise cancelling applied to the EEG," IEEE Trans. Biomed. Eng., vol. 44, pp. 775-779, Aug. 1997.

[3] B. Van Veen, J. Joseph, and K. Hecox, "Localization of intra-cerebral sources of electrical activity via linearly constrained minimum variance spatial filtering," IEEE Signal Processing Workshop Statistics and Signal Array Processing, 1992, pp. 526-529.

[4] M. E. Spencer, R. M. Leahy, J. C. Mosher, and P. S. Lewis, "Adaptive filters for monitoring localized brain activity from surface potential time series," Proc. 26th Asilomar Conf. Signal Syst. Comp., vol. 1, pp. 156-161, 1992.

[5] W. van Drongelen, M. Yuchtman, B. Van Veen, and A. van Huffelen, "A spatial filtering technique to detect and localize multiple sources in the brain," Brain Topogr., vol. 9, no. 1, pp. 39-49, 1996.

[6] B. D. Van Veen and K. M. Buckley, "Beamforming: A versatile approach to spatial filtering," IEEE Acoust., Speech, Signal Processing, Mag., vol. 5, pp. 4-24, 1988.

[7] L. J. Griffiths and C. W. Jim, "An alternative approach to linearly constrained adaptive beamforming," IEEE Trans. Antennas Propagat., vol. AP-30, pp. 27-34, 1982.

[8] H. H. Jasper, "Report of the committee on methods of clinical examination in electroencephalography," Electroencephal. Clin. Neurophysiol., vol. 10 , pp. $370-375,1958$.

[9] Y. Salu, L. G. Cohen, D. Rose, S. Sato, C. Kufta, and M. Hallett, "An improved method for localizing electric brain dipoles," IEEE Trans. Biomed. Eng., vol. 37, pp. 699-705, July 1990.

[10] S. Rush and D. A. Driscoll, "EEG electrode sensitivity-An application of reciprocity," IEEE Trans. Biomed. Eng., vol. BME-16, pp. 15-22, Jan. 1969.

[11] B. N. Cuffin, "Effects of local variations in skull and scalp thickness on EEG's and MEG's," IEEE Trans. Biomed. Eng., vol. 40, pp. 42-48, Jan. 1993.

[12] B. J. Roth, M. Balish, A. Gorbach, and S. Sato, "How well does a three-sphere model predict positions of dipoles in realistically shaped heads?," Electroencephal. Clin. Neurophysiol., vol. 87, pp. 175-184, 1993.

[13] A. A. Dingle, R. D. Jones, G. J. Carroll, and W. R. Fright, "A multistage system to detect epileptiform activity in the EEG," IEEE Trans. Biomed. Eng., vol. 40, pp. 1260-1268, Dec. 1993.

[14] R. D. Jones, A. A. Dingle, G. J. Carroll, R. D. Green, M. A. Black, I. M. Donaldson, P. J. Parkin, P. J. Bones, and K. L. Burgess, "A system for detecting epileptiform activity in the EEG: Real-time operation and clinical trial," Proc. Ann. Int. Conf. IEEE Eng. Med. Biol. Soc., 1996, vol. 18, pp. 948-949. [Online]. Available: CD-ROM.

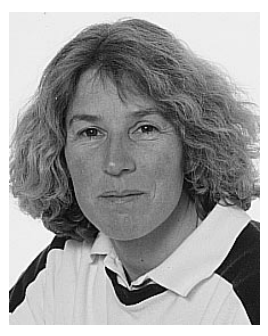

Donna-Maree Ward (S'93-M'97) received the B.Sc. degree in physics mathematics in 1987 , the M.Sc. (Hons.) degree in astronomy in 1992, and the Ph.D. degree in electrical and electronic engineering in 1998 from the University of Canterbury, Christchurch, New Zealand. Her Ph.D. research involved investigating an adaptive "beamforming" technique to noninvasively enhance epileptiform activity from deep foci in the brain.

She is currently a software design engineer involved in the development of a fully integrated real-time Treasury Management System. Her research interest is primarily concerned with the application of adaptive signal processing techniques to EEG signal analysis, with particular emphasis on detecting and enhancing epileptic activity. 


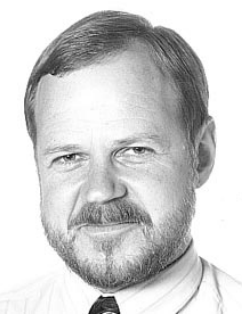

Richard D. Jones (M'87-SM'90) received the B.E. (Hons.) and M.E. degrees in electrical and electronic engineering from the University of Canterbury, Christchurch, New Zealand, in 1974 and 1975, respectively, and the Ph.D. degree in medicine from the Christchurch School of Medicine, University of Otago, Christchurch, in 1987.

$\mathrm{He}$ is a Biomedical Engineer and Neuroscientist, Head of the Diagnostic Physics and Bioengineering Section in the Department of Medical Physics and Bioengineering at Christchurch Hospital and a member of the Department of Medicine at the Christchurch School of Medicine, University of Otago, and a member of the Faculty of Engineering at the University of Canterbury. His research interests include: 1) human performance engineering - development and application of the computerized tests for quantification of upper-limb sensory-motor function, particularly in brain disorders (stroke, Parkinson's disease) and driving assessment; 2) signal processing in clinical neurophysiology-real-time EEG analysis for detection of epileptic activity, spectral topography, and long-term EEG monitoring; and 3) eye movements in brain disorders.

Dr. Jones is a Registered Engineer, a Fellow of the Institution of Professional Engineers New Zealand, a Fellow and Immediate Past President of the Australiasian College of Physical Scientists and Engineers in Medicine. He was Representative for the Asis/Pacific Region on the Administrative Committee of the IEEE Engineering in Medicine and Biology Society in 1993/1994, has been a member of the EMBS', International Program Committee since 1988, and is an Associate Editor of IEEE Transactions ON BIOMEDICAL ENGINEERING.

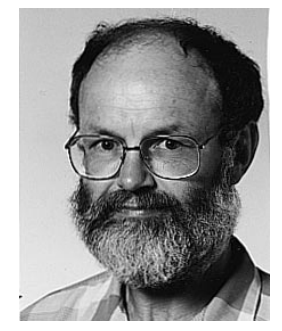

Philip J. Bones (S'81-M'82-SM'91) was born in 1951 in Palmerston North, New Zealand. He received the B.E. (Hons), M.E., and Ph.D. degrees in electrical and electronic engineering from the University of Canterbury, Christchurch, New Zealand, in 1973,1975 , and 1981 , respectively.

He worked for 11 years as Biomedical Engineer in the Department of Cardiology, Christchurch, and an additional two as Postdoctoral Fellow with cardiac groups in Europe (including one year as Alexander von Humboldt fellow in Heidelberg, Germany). Since 1988 he has been Senior Lecturer in the Department of Electrical and Electronic Engineering, University of Canterbury, Christchurch, New Zealand. His research interests include the application of signal processing techniques to physiological signals, medical imaging, and problems in image recovery.

Dr. Bones is a member of S.P.I.E. and the Australasian College of Physical Scientists and Engineers in Medicine.

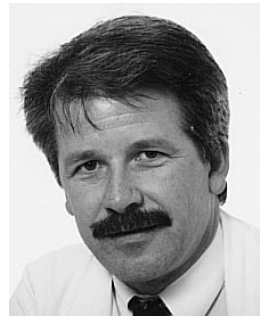

Grant J. Carroll received the B.Sc. degree in physiology and the Post-Graduate Diploma in Science and Physiology from the University of Otago, Dunedin, New Zealand, in 1976 and 1977, respectively. He then underwent training in clinical neurophysiology at Auckland Hospital, Auckland.

Since 1979, he has been Clinical Neurophysiologist in the Department of Neurology, Christchurch Hospital, Christchurch, New Zealand. His main research interests include the electrophysiological diagnosis of peripheral neuropathies, the electrophysiological assessment of pelvic floor function, and the computer-based analysis of neurophysiological recordings.

Mr. Carroll is a member of the Neurological Association of New Zealand. 